\title{
Algebraic analysis in structures with the Kaplansky-Jacobson property
}

\author{
by \\ D. Przeworska-Rolewicz (Warszawa)
}

\begin{abstract}
In $1950 \mathrm{~N}$. Jacobson proved that if $u$ is an element of a ring with unit such that $u$ has more than one right inverse, then it has infinitely many right inverses. He also mentioned that I. Kaplansky proved this in another way. Recently, K. P. Shum and Y. Q. Gao gave a new (non-constructive) proof of the Kaplansky-Jacobson theorem for monoids admitting a ring structure. We generalize that theorem to monoids without any ring structure and we show the consequences of the generalized Kaplansky-Jacobson theorem for the theory of linear operators, and even for the classical Calculus. In order to do that, we recall some multiplicative systems, called pseudocategories, very useful in the algebraic theory of perturbations of linear operators. In the second part of the paper, basing on the Kaplansky-Jacobson theorem, we show how to use the above mentioned structures for building Algebraic Analysis of linear operators over a class of linear spaces. We also define (non-linear) logarithmic and antilogarithmic mappings on these structures.
\end{abstract}

In 1950 N. Jacobson proved the following theorem:

If $u$ is an element of a ring with unit e such that $u$ has more than one right inverse, then it has infinitely many right inverses (cf. [J1]).

Namely, all elements of the form $w_{k}=v_{0}+\left(e-v_{0} u\right) u^{k}(k \in \mathbb{N})$ are also right inverses of $u$, for any fixed right inverse $v_{0}$.

In the same paper [J1] it is mentioned that I. Kaplansky proved this theorem in another way (private communication to Jacobson).

Recently, K. P. Shum and Y. Q. Gao gave a new (non-constructive) proof of the Kaplansky-Jacobson theorem for monoids admitting a ring structure (cf. [SG], also [B]). In this paper we generalize that theorem to monoids without any ring structure and we show the deep consequences of the gener-

2000 Mathematics Subject Classification: 44A40, 16Y99, 20M10, 33B99, 47A05, 47A55, 47A60, 47D99.

Key words and phrases: pseudocategory, pararing, paraalgebra, right (left) inverse, index of linear operator, perturbation of linear operators, algebraic analysis, logarithmic mapping, antilogarithmic mapping, Leibniz condition. 
alized Kaplansky-Jacobson theorem for the theory of linear operators, and even for the classical Calculus.

In order to do that, we will recall some multiplicative systems, called pseudocategories, very useful in the algebraic theory of perturbations of linear operators. These were introduced in the monograph of the present author and S. Rolewicz ([PRR1]), where pararings and paraalgebras were defined for operators mapping a linear space into another. An axiomatics of pseudocategories was given in [PR1]. Next, in [PR2], in a rather complicated way, properties of pseudocategories were studied. It was shown that pseudocategories can be extended to concrete categories under some additional assumptions. Furthermore pararings and paraalgebras, ideals and radicals were defined by means of pseudocategories. Then, as in the above-mentioned book, the algebraic theory of perturbations of linear operators over a class of linear spaces was examined. This theory was simplified in an essential way by H. Lausch in 1985 (cf. [LPR]).

It should be pointed out that in the theory of pseudocategories the notion of objects does not play any role. The axiomatics contains only the notion of morphisms. The existence of a unit is also not necessary. This is natural in a sense. For instance, in a quotient paraalgebra of linear operators over a class of linear spaces, the spaces under consideration are not objects. One can introduce objects in this case, but in an artificial way.

In the second part of the present paper, due to the Kaplansky-Jacobson theorem, it will be shown how to use the above mentioned structures for building Algebraic Analysis of linear operators over a class of linear spaces. We shall also define (non-linear) logarithmic and antilogarithmic mappings on these structures.

\section{Pseudocategories, pararings, paraalgebras and perturbations} of linear operators. We recall some notions and properties (without proofs which can found in $[\mathrm{LPR}]$ ) which will be used in what follows.

1a. Pseudocategories. The following definition is fundamental for all subsequent considerations:

Definition 1a.1. A class $P$ of morphisms is called a pseudocategory if for some ordered pairs $(x, y)$ of morphisms $x, y \in P$ a product $z \in P$ is defined, denoted by $z=x y$, such that for all $a, b, c, d \in P$ the following axioms are satisfied:

$\mathbf{A}_{1}: \quad(a b, c d, c b$ exists $) \Rightarrow a d$ exists;

$\mathbf{A}_{2}:(a b, b c$ exist $) \Rightarrow[(a b) c, a(b c)$ exist and $(a b) c=a(b c)]$; call the last element $a b c$; 
$\mathbf{A}_{3}: \quad[\forall a(a b$ exists $\Rightarrow(a b) c$ exists $)] \Rightarrow b c$ exists;

$\left[\forall b(b c\right.$ exists $\Rightarrow a(b c)$ exists $) \Rightarrow a b$ exists $\left({ }^{1}\right)$;

$\mathbf{A}_{4}:\{x \in P: a x, x b$ exist $\}$ is a set.

Definition 1a.2. If $P$ is a pseudocategory and $a, b \in P$ then $\{x \in P$ : $a x, x b$ exist $\}$ is called a multiplicant and is denoted by $M_{a \mid b}$.

Proposition 1a.1. Let $P$ be a pseudocategory.

(i) If $a, \widetilde{a}, b, \widetilde{b}, x, y \in P$ and $a x, \widetilde{a} x, y b, y \widetilde{b}$ exist then $M_{\widetilde{a} \mid \widetilde{b}}=M_{a \mid b}$;

(ii) if $c, d \in P$ and $M_{c \mid c} \cap M_{d \mid d} \neq \emptyset$ then $M_{c \mid c}=M_{d \mid d}$;

(iii) if $c, u, v \in P$ and $u v$ exists then $M_{c \mid u}=M_{c \mid u v}$ and $M_{v \mid c}=M_{u v \mid c}$;

(iv) if $a, x, y, z \in P$ and $x, y \in M_{a \mid a}$ then the existence of $z x$ implies the existence of $z y$ and the existence of $x z$ implies the existence of $y z$.

Definition 1a.3. A subclass $Q$ of a pseudocategory $P$ is a subpseudocategory if whenever $x, y \in P$ and $x y$ exists then $x y \in Q$.

Note that any subpseudocategory $Q$ of a pseudocategory $P$, equipped with the multiplication of $P$, is itself a pseudocategory.

Proposition 1a.2. Let $P$ be a pseudocategory and let $a \in P$. Then both

$$
P_{a}=\left\{x \in P: M_{a \mid x} \neq \emptyset\right\} \quad \text { and } \quad P_{a}^{\prime}=\left\{y \in P: M_{y \mid a} \neq \emptyset\right\}
$$

are subpseudocategories of $P$.

Definition 1a.4. A pseudocategory $P$ is proper if $M_{a \mid a} \neq \emptyset$ for all $a \in P$.

THEOREM 1a.1. If $P$ is a proper pseudocategory then

(i) for all $a, b \in P$ either $M_{a \mid b}=\emptyset$ or there exists a $y \in P$ such that $M_{a \mid b}=M_{y \mid y}$

(ii) $P=\bigcup_{y \in P} M_{y \mid y}$, i.e. the proper pseudocategory is the union of disjoint multiplicants of the form $M_{y \mid y}$, where $y \in P$.

Proposition 1a.3. Let $P$ be a proper pseudocategory and let $a \in P$. Then $a \cdot a=a^{2}$ exists if and only if $a \in M_{a \mid a}$. If $a \in M_{a \mid a}$ then $M_{a \mid a}$ is a semigroup.

Definition 1a.5. Let $P$ be a pseudocategory and let $a \in P$ have the property that $a \in M_{a \mid a}$. A morphism $e_{a} \in M_{a \mid a}$ is called a unit in $P$ if

$$
\forall x \in P\left(x e_{a} \text { exists } \Rightarrow x e_{a}=x \text { and } e_{a} x \text { exists } \Rightarrow e_{a} x=x\right) .
$$

We say that $P$ has units if each multiplicant $M_{a \mid a}$ with $a \in M_{a \mid a}$ contains a unit.

$\left({ }^{1}\right)$ This axiom is written here in a slightly modified form in comparison with [LPR]. 
TheOREM 1a.2. Let $P$ be a proper pseudocategory. Then there exists a proper pseudocategory $P_{1}$ with units such that $P$ is its subpseudocategory.

COROLlary 1a.1. Every proper pseudocategory is a subpseudocategory of some category.

Definition 1a.6. Two disjoint subclasses $Q$ and $R$ of a pseudocategory $P$ are said to be non-cooperating if $y \in Q, z \in R$ implies that neither $y z$ nor $z y$ exist. A proper pseudocategory $P$ is said to be irreducible if $P$ has no two non-empty non-cooperating subpseudocategories (with respect to the same multiplication).

Proposition 1a.4. Let $P$ be an irreducible pseudocategory and let $a, b \in P$. Then $M_{a \mid b} \neq \emptyset$.

Definition 1a.7. A pseudocategory $P$ is said to be indexed if there exists a set $\mathrm{A}$ and a map $\mathrm{M}: \mathrm{A} \times \mathrm{A} \rightarrow\left\{M_{u \mid u}: u \in P\right\},(\alpha, \beta) \mapsto M_{\alpha, \beta}$, such that

(i) $M_{\alpha, \beta} \cap M_{\alpha^{\prime}, \beta^{\prime}}=\emptyset$ if $(\alpha, \beta) \neq\left(\alpha^{\prime}, \beta^{\prime}\right)$;

(ii) $\mathrm{M}$ is surjective;

(iii) $x \in M_{\alpha, \beta}, y \in M_{\beta, \gamma}(\alpha, \beta, \gamma \in \mathrm{A})$ imply that $x y$ exists and $x y \in M_{\alpha, \gamma}$.

$\mathrm{M}$ is called an indexing of $P$.

TheOREM 1a.3. If an irreducible pseudocategory $P$ is a set then it is indexed.

Definition 1a.8. Let $\mathfrak{M}$ be a class of sets and $P$ be a class of mappings between sets of $\mathfrak{M}$. If $P$ is a pseudocategory with respect to composition of mappings then $P$ is called a concrete pseudocategory.

TheOREM 1a.4. Let $P$ be a pseudocategory indexed by $\mathrm{M}: \mathrm{A} \times \mathrm{A} \rightarrow$ $\left\{M_{u \mid u}: u \in P\right\}$. Then $P$ is isomorphic (in the obvious sense) to a concrete pseudocategory with the same indexing $\mathrm{M}$.

A proper pseudocategory is said to be commutative if whenever $a b$ exists then $b a$ exists and $b a=a b$.

An irreducible pseudocategory which is a set is a semigroup if

$$
a b \text { exists } \Rightarrow b a \text { exists. }
$$

Let $P$ be a proper pseudocategory with units and let $x \in P$. If there exist $x^{l} \in P$ (respectively, $x^{r} \in P$ ) such that for some $a \in M_{a \mid a}$ we have $x^{l} x=e_{a}$ (respectively, $x x^{r}=e_{a}$ ) then $x$ is said to be left invertible (respectively, right invertible). The morphisms $x^{l}, x^{r}$ are called left and right inverses of $x$, respectively. 
Proposition 1a.5. If an $x$ belonging to a proper pseudocategory with units is simultaneously left and right invertible then its left inverse is equal to its right inverse.

A morphism $x$ satisfying the conditions of Proposition 1a.5 is said to be invertible and we write $x^{-1}=x^{l}=x^{r}$. The morphism $x^{-1}$ is called an inverse. The inverse of every invertible morphism is, by definition, uniquely determined and $x^{-1} \in M_{x \mid x}$ (for both $x x^{-1}$ and $x^{-1} x$ exist).

A proper pseudocategory $P$ with units is said to be a paragroup if every $x \in P$ is invertible. An irreducible paragroup which is a set satisfying condition (1a.1) is a group.

It is easy to verify that if $P$ is an irreducible pseudocategory then the class of all multiplicants contained in $P$ with multiplication defined as follows is a Brandt grupoid:

(i) if $a \in M_{a \mid a}$ then $M_{a \mid a} \cdot M_{a \mid a}=M_{a \mid a}$;

(ii) if $a \notin M_{a \mid a}$ and $b \in M_{a \mid a}$ then $M_{a \mid a} \cdot M_{b \mid b}=M_{b a \mid b a} ; M_{b \mid b} \cdot M_{a \mid a}=$ $M_{a b \mid a b} ; M_{a \mid a} \cdot M_{a b \mid a b}=M_{b a \mid b a} \cdot M_{a \mid a}=M_{a \mid a} ; M_{a b \mid a b} \cdot M_{b \mid b}=$ $M_{b \mid b} \cdot M_{b a \mid b a}=M_{b, b}$ (cf. H. Brandt [Bra], also R. H. Bruck [Bru]).

1b. Pararings. We start with

Definition 1b.1. A pararing $P$ is a proper pseudocategory such that for each $a \in P$ an addition + is defined on $M_{a \mid a}$ which turns $M_{a \mid a}$ into an Abelian group and this addition satisfies

$\mathbf{A}_{5}: \quad$ if $x, y \in M_{a \mid a}, z \in P$, then

$$
z x \text { exists } \Rightarrow z(x+y)=z x+z y ; \quad x z \text { exists } \Rightarrow(x+y) z=x z+y z .
$$

Note that, by Proposition 1a.1(iv), the existence of $z x$ (respectively, $x z$ ) implies the existence of $z y$ and $z(x+y)$ (respectively $y z$ and $(x+y) z$ ). Hence the right hand sides in $\mathbf{A}_{5}$ are always defined.

Definition 1b.2. A subclass $P_{0}$ of a pararing $P$ is said to be a subpararing of $P$ if $P_{0}$ is a pararing with the addition and multiplication of $P$ restricted to $P_{0}$. A subpararing $J$ of a pararing $P$ is an ideal of $P$ if for all $x \in P$ and $z \in J$ the existence of $x z$ implies that $x z \in J$ and the existence of $z x$ implies that $z x \in J$. If $a \in P$ and $x \in M_{a \mid a}$ then $[x]=\{x+y: y \in$ $\left.M_{a \mid a} \cap J\right\}$ is called the coset (with respect to $J$ ) determined by $x$.

Note that $[x] \subseteq M_{a \mid a} \cap J$ for $x \in M_{a \mid a}$.

Definition 1b.3. Let $A, B$ be subsets of a pararing $P$. We write

$$
\begin{aligned}
A+B & =\{x+y: x \in A, y \in B, x+y \text { exists }\}, \\
A B & =\{x y: x \in A, y \in B, x y \text { exists }\} .
\end{aligned}
$$


Theorem 1b.1. Let $J$ be an ideal in a pararing $P$ and let $x_{1}, x_{2}, x_{3} \in P$. Suppose that $x_{1}+x_{2}$ and $x_{1} x_{3}$ exist. Then $\left[x_{1}\right]+\left[x_{2}\right]=\left[x_{1}+x_{2}\right]$ and $\left[x_{1}\right]\left[x_{3}\right] \subseteq\left[x_{1} x_{3}\right]$.

Definition 1b.4. Let $J$ be an ideal of a pararing $P$. The quotient pararing $P / J$ is the class of all cosets $[x]$ with respect to $J$, for $x \in P$, with addition and multiplication defined in the usual way.

Theorem 1b.1 tells us that $P / J$ is a pararing and $[x][y]$ exists if and only if $x y$ exists.

Theorem 1b.2. Suppose that $P$ is a pararing and $a \in P$. Denote by $0_{a}$ the neutral morphism of the multiplicant $M_{a \mid a}\left(^{1}\right)$ and by $N(P)$ the class of neutral morphisms belonging to $P$. Then

(i) if $a \in M_{a \mid a}$ then $M_{a \mid a}$ is a ring;

(ii) if $a \in P$ then $M_{a x \mid a x}$ and $M_{x a \mid x a}$ are independent of the choice of $x \in M_{a \mid a}$;

(iii) if $a \in P$ and $x \in M_{a \mid a}$ then $M_{a \mid a}$ is a right (left) module $\left({ }^{2}\right)$ over the ring $M_{a x \mid a x}$ (respectively, $M_{x a \mid x a}$ ); moreover,

$$
0_{x a} x=0_{a}, \quad x 0_{a x}=0_{a} .
$$

A pararing $P$ has units if $P$ considered as a pseudocategory has units. A pararing with units is said to be a parafield if every non-neutral morphism is invertible. Evidently, an irreducible parafield which is a set is a field.

ExAmple 1b.1. Let $X$ and $Y$ be linear spaces over the same field of scalars. Denote by $L_{0}(X \rightarrow Y)$ the space of all linear operators defined on $X$ and with range in $Y$. Write $L_{0}(X)=L_{0}(X \rightarrow X)$. The quadruple

$$
L_{0}(X \rightleftharpoons Y)=\left(\begin{array}{ccc}
L_{0}(X \rightarrow Y) & L_{0}(X) & \\
& L_{0}(Y) & L_{0}(Y \rightarrow X)
\end{array}\right)
$$

is a pararing which has been considered by the present author and S. Rolewicz in [PRR1]) (cf. also Example 1c.1).

One can prove that every pararing can be extended to an additive pseudocategory. However, this extension seems to be rather artificial (cf. [PR2]). On the other hand, there is a pararing which is not an additive category, for instance, the ring $\mathbb{Z}$ of all integers.

Definition 1b.5. Let $P$ be a pararing with units. The subclass

$$
\mathbf{R}(P)=\left\{x \in P: \forall a, y, z \in P\left[a \in M_{a \mid a} \text { and } y x z \in M_{a, a}\right]\right.
$$

is called the radical of $P$.

$$
\left.\Rightarrow e_{a}+y x z \text { is invertible }\right\}
$$

\footnotetext{
$\left({ }^{1}\right)$ That is, a morphism in $M_{a \mid a}$ such that $0_{a} x=x 0_{a}=0_{a}$ for all $x \in P$.

$\left({ }^{2}\right)$ See Jacobson [J2].
} 
TheOREM 1b.3. The radical $\mathbf{R}(P)$ of a pararing $P$ with units is an ideal in $P$.

Open QUESTIONS. Does every non-commutative pararing without divisors of neutral morphisms have an extension to a parafield? Is every ideal in a pararing with units contained in a maximal ideal? Is the radical of a pararing with units an intersection of maximal ideals?

1c. Paraalgebras and perturbations of linear operators

Definition 1c.1. A pararing $P$ with units is said to be a paraalgebra if

(i) every multiplicant $M_{a \mid a}$ is a linear space over a field $\mathbb{F}$ of scalars;

(ii) whenever $x y$ exists then $t(x y)=(t x) y=x(t y)$ for $t \in \mathbb{F}$.

EXAmple 1c.1. Suppose that a class $\left\{X_{\alpha}\right\}_{\alpha \in \mathbf{A}}$ of linear spaces (over a field $\mathbb{F}$ of scalars) is given. It is easy to verify that

$$
L_{0}\left(X_{\alpha} \rightleftharpoons X_{\beta} ; \alpha, \beta \in \mathbf{A}\right)=\bigcup_{\alpha, \beta \in \mathbf{A}} L_{0}\left(X_{\alpha} \rightarrow X_{\beta}\right)
$$

is a paraalgebra. If $\mathbf{A}$ is a set then this paraalgebra is indexed by $\mathbf{A}$. The spaces $L_{0}\left(X_{\alpha} \rightarrow X_{\beta}\right)$ are multiplicants, $L_{0}\left(X_{\alpha} \rightarrow X_{\alpha}\right)$ are multiplicants such that $T^{2}=T \cdot T \in L_{0}\left(X_{\alpha} \rightarrow X_{\alpha}\right)$ whenever $T \in L_{0}\left(X_{\alpha} \rightarrow X_{\alpha}\right)$. The paraalgebra $L_{0}\left(X_{\alpha} \rightleftharpoons X_{\beta} ; \alpha, \beta \in \mathbf{A}\right)$ has units, namely the identity operators $I_{\alpha}$ of the spaces $X_{\alpha}$ (cf. Example 1b.1).

EXAMPLE 1c.2. Let $\mathcal{L}_{T}$ be the class of all linear topological spaces (over $\mathbb{C}$ or $\mathbb{R})$. The class $L T\left(X \rightleftharpoons Y ; X, Y \in \mathcal{L}_{T}\right)$ of all linear continuous operators defined on spaces $X \in \mathcal{L}_{T}$ and mapping $X$ into spaces belonging to $\mathcal{L}_{T}$ is a paraalgebra which is not a set.

Note that Banach paraalgebras have been considered by K. H. Förster (cf. $[\mathrm{F}]$ ).

EXAMPLE 1c.3. Let $\mathcal{E}\left(\mathbb{E}^{m} \rightleftharpoons \mathbb{E}^{n} ; m, n \in \mathbb{N}\right)$ be the paraalgebra of all $m \times n$ matrices with coefficients from a field $\mathbb{E}$ of scalars. If $m \neq n$ then a matrix $U \in \mathcal{E}\left(\mathbb{E}^{m} \rightleftharpoons \mathbb{E}^{n} ; m, n \in \mathbb{N}\right)$ is not invertible. This implies the following fact: if $L_{0}\left(X_{\alpha} \rightleftharpoons X_{\beta} ; \alpha, \beta \in \mathbf{A}\right)$ is a paraalgebra defined in Example 1c.1 and $\operatorname{dim} X_{\alpha}<\infty$ for all $\alpha \in \mathbf{A}$ then an operator $T \in L_{0}\left(X_{\alpha} \rightleftharpoons X_{\beta} ; \alpha, \beta \in \mathbf{A}\right)$ is invertible if and only if $T \in L_{0}\left(X_{\alpha} \rightarrow X_{\alpha}\right)$.

Let $J$ be an ideal in a paraalgebra $P$ and let $a \in P$. An element $r$ is called a left (right) regularizer to the ideal $J$ if $r a$ (respectively, ar) exists and, for some $c \in P$ such that $c \in M_{c \mid c}, r a \in M_{c \mid c}$ (respectively, ar $\in M_{c \mid c}$ ) and $r a=e_{c}+b$ (respectively, $a r=e_{c}+b$ ), where $b \in J \cap M_{c \mid c}$. This means that in the quotient paraalgebra $P / J$ the corresponding coset is left (right) invertible. If $r$ is simultaneously a left and right regularizer then $r$ is called a simple regularizer and the corresponding coset is invertible. 
Let $A$ be a linear operator defined on a linear subset $\operatorname{dom} A$ of a linear space $X$, called the domain of $A$, and mapping dom $A$ into a linear space $Y$ (both over the same field $\mathbb{F}$ of scalars). The set of all such operators will be denoted by $L(X \rightarrow Y)$. Write

$\alpha_{A}=\operatorname{dim} \operatorname{ker} A=\operatorname{dim}\{x \in \operatorname{dom} A: A x=0\}, \beta_{A}=\operatorname{codim} E_{A}=\operatorname{dim} Y / E_{A}$, where $E_{A}=A(\operatorname{dom} A) \subset Y$ is the range of $A$; and

$$
\kappa_{A}= \begin{cases}-\infty & \text { if } \alpha_{A}=\infty, \beta_{A}<\infty \\ \infty & \text { if } \alpha_{A}<\infty, \beta_{A}=\infty \\ \beta_{A}-\alpha_{A} & \text { if } \alpha_{A}, \beta_{A}<\infty\end{cases}
$$

The numbers $\alpha_{A}, \beta_{A}, \kappa_{A}$ are called the nullity, deficiency and index of the operator $A \in L(X \rightarrow Y)$, respectively. The ordered pair $\left(\alpha_{A}, \beta_{A}\right)$ is called the $d$-characteristic (dimensional characteristic) of $A$ (cf. [PRR1]). If both $\alpha_{A}$ and $\beta_{A}$ are finite then the $d$-characteristic is said to be finite.

Suppose that $\mathcal{X}$ is a class of linear spaces (over the same field of scalars). Let $L(X \rightleftharpoons Y ; \mathcal{X})$ be the paraalgebra of linear operators mapping spaces belonging to $\mathcal{X}$ into spaces from $\mathcal{X}$. This paraalgebra has units, namely, the identity operators $I_{X}$ of spaces $X \in \mathcal{X}$.

If every operator $A \in L(X \rightleftharpoons Y$; $\mathcal{X})$ with a finite $d$-characteristic has a simple regularizer to an ideal $J \subset L(X \rightleftharpoons Y ; \mathcal{X})$ then we say that $L(X \rightleftharpoons Y ; \mathcal{X})$ is regularizable to the ideal $J$. The paraalgebra $L_{0}\left(X_{\alpha} \rightleftharpoons X_{\beta}\right.$; $\alpha, \beta \in \mathbf{A})$ is regularizable to the ideal of all finite-dimensional operators contained in this paraalgebra.

An ideal $J$ in a paraalgebra $L(X \rightleftharpoons Y ; \mathcal{X})$ is said to be a quasi Fredholm ideal if for all $X \in \mathcal{X}$ and for all $T \in J \cap L_{0}(X \rightarrow X)$ the operator $I_{X}+T$ has a finite $d$-characteristic. If, moreover, $\kappa_{I_{X}+T}=0$ then $J$ is said to be a Fredholm ideal.

TheOREM 1c.1. Suppose that a paraalgebra $\mathcal{L}_{\mathcal{X}}=L(X \rightleftharpoons Y ; \mathcal{X})$ is regularizable to a quasi Fredholm ideal in $J \subset \mathcal{L}_{\mathcal{X}}$. Denote by $\mathbf{R}\left(\mathcal{L}_{\mathcal{X}} / J\right)$ the radical of the quotient paraalgebra $\mathcal{L}_{\mathcal{X}} / J$. Then the ideal $J_{0}=\left\{U \in \mathcal{L}_{\mathcal{X}}\right.$ : $\left.[U] \in \mathbf{R}\left(\mathcal{L}_{\mathcal{X}} / J\right)\right\}$ is the maximal quasi Fredholm ideal, i.e. $J \subset J_{0}$.

An ideal $J$ in a paraalgebra $\mathcal{L}_{\mathcal{X}}=L(X \rightleftharpoons Y ; \mathcal{X})$ is called a positive (negative) semi-Fredholm ideal if for all $X \in \mathcal{X}$ and $T \in J \cap L_{0}(X \rightarrow X)$ the operator $I_{X}+T$ has a finite nullity (deficiency). A paraalgebra $\mathcal{L}_{\mathcal{X}}=$ $L(X \rightleftharpoons Y ; \mathcal{X})$ is called left (right) regularizable to an ideal $J \subset \mathcal{L}_{\mathcal{X}}$ if every operator $\in \mathcal{L}_{\mathcal{X}}$ with a finite nullity (deficiency) has a left (right) regularizer to the ideal $J$.

Let $\mathfrak{A}$ be a class (not necessarily linear) of linear operators. A linear operator $B$ is called a perturbation of an operator $A \in \mathfrak{A}$ if $A+B \in \mathfrak{A}$. $B$ is 
said to be a perturbation of the class $\mathfrak{A}$ if $A+B \in \mathfrak{A}$ for all $A \in \mathfrak{A}$ such that the sum $A+B$ is well defined.

TheOREM 1c.2. Suppose that a paraalgebra $\mathcal{L}_{\mathcal{X}}=L(X \rightleftharpoons Y ; \mathcal{X})$ is left (right) regularizable to a positive (negative) semi-Fredholm ideal $J \subset \mathcal{L}_{\mathcal{X}}$. Then all operators belonging to a positive (negative) semi-Fredholm ideal $J_{1} \subset \mathcal{L}_{\mathcal{X}}$ are perturbations of the class of all operators with a finite nullity (deficiency) belonging to $\mathcal{L}_{\mathcal{X}}$.

Corollary 1c.1. If a paraalgebra $\mathcal{L}_{\mathcal{X}}=L(X \rightleftharpoons Y ; \mathcal{X})$ is regularizable to a quasi Fredholm ideal $J \subset \mathcal{L}_{\mathcal{X}}$ then every $T \in J$ is a perturbation of the class of all operators with a finite d-characteristic. If $J$ is a Fredholm ideal then this perturbation preserves the index, i.e. for all $A \in \mathcal{L}_{\mathcal{X}}$ with a finite $d$-characteristic and for all $T \in J$ such the sum $A+T$ is well defined, we have $\kappa_{A+T}=\kappa_{A}$.

Regularizers of integral and singular integral operators have been in common use for several years (cf. for instance Nguyen Van Mau [N1], [N2]).

2. Pararings with the Kaplansky-Jacobson property. We start with

Theorem 2.1. Let $P$ be a pararing with units. Let $x \in P$ be right invertible and let $x^{r}$ be its right inverse. Then there is an $a \in P$ such that the morphism $p_{x}=e_{a}-x^{r} x$ is well defined and $p_{x} \in M_{a \mid a}$. Moreover, $p_{x}$ is an idempotent such that $p_{x} x^{r}=0$ and $x p_{x}=0\left(^{1}\right)$.

Proof. By definition, there is an $a \in P$ such that $a \in M_{a \mid a}$ and $x x^{r}=e_{a}$. By Proposition 1a.1(iv), $x^{r} x$ exists and belongs to $M_{a \mid a}$. Thus the morphism $p_{x}=e_{a}-x^{r} x$ is well defined and belongs to $M_{a \mid a}$. By Proposition 1a.3, $p_{x}^{2}$ exists, belongs to $M_{a \mid a}$ and

$$
\begin{aligned}
p_{x}^{2} & =\left(e_{a}-x^{r} x\right)^{2}=\left(e_{a}-x^{r} x\right)\left(e_{a}-x^{r} x\right) \\
& =e_{a}^{2}-\left(x^{r} x\right) e_{a}-e_{a}\left(x^{r} x\right)+\left(x^{r} x\right)\left(x^{r} x\right) \\
& =e_{a}-\left(e_{a} x^{r}\right) x-x^{r}\left(x e_{a}\right)+x^{r}\left(x x^{r}\right) x \\
& =e_{a}-x^{r} x-x^{r} x+x^{r} e_{a} x=e_{a}-x^{r} x=p_{x},
\end{aligned}
$$

i.e. $p_{x}$ is an idempotent. Moreover, $x p_{x}=x\left(e_{a}-x^{r} x\right)=x e_{a}-\left(x x^{r}\right) x=$ $x-e_{a} x=0$ and $p_{x} x^{r}=\left(e_{a}-x^{r} x\right) x^{r}=e_{a} x^{r}-x^{r}\left(x x^{r}\right)=x^{r}-x^{r} e_{a}=0$.

Corollary 2.1. Let $P$ be a pararing with units. Let $x \in P$ be right invertible and let $x^{r}$ be its right inverse, i.e. there is an $a \in P$ such that the idempotent $p_{x}=e_{a}-x^{r} x \in M_{a \mid a}$. Then every right inverse of $x$ is of the

$\left({ }^{1}\right)$ Here and in what follows we denote all neutral morphisms $0_{a}$ in $P$ by 0 , since this does not lead to any misunderstanding. 
form $x_{1}^{r}=x^{r}+p_{x} y$, where $p_{x} y$ exists and belongs to $M_{a \mid a}$. In particular, $x_{k}^{r}=x^{r}+p_{x} x^{k}$ for $k \in \mathbb{N}$ are right inverses of $x\left(^{1}\right)$.

Proof. By Theorem 2.1, if $p_{x} y$ exists and belongs to $M_{a \mid a}$ then $x x_{1}^{r}=$ $x\left(x^{r}+p_{x} y\right)=x x^{r}+\left(x p_{x}\right) y=e_{a}+0=e_{a}$, i.e. $x_{1}^{r}$ is a right inverse of $x$. A similar proof for $x_{k}^{r}$, since $x^{r} x$ and $x^{k}$ exist.

TheOREM 2.2. Let $P$ be a pararing with units. Let $x \in P$ be right invertible and let $p_{x}=e_{a}-x^{r} x \in M_{a \mid a}$ be an idempotent corresponding to a right inverse $x^{r}$ of $x\left(a \in M_{a \mid a} \subset P\right)$. Whenever $y x$ exists and belongs to $M_{a \mid a}$, the morphism $\widetilde{p}_{x}=p_{x}\left(e_{a}-y x\right)$ is an idempotent corresponding to a right inverse $\widetilde{x}^{r}=x^{r}-\widetilde{p}_{x} x^{r}$ of $x$ such that $x \widetilde{p}_{x}=0$ and $\widetilde{p}_{x} \widetilde{x}^{r}=0$.

Proof. By definitions,

$$
\begin{gathered}
\widetilde{p}_{x} \widetilde{x}^{r}=\widetilde{p}_{x}\left(x^{r}-\widetilde{p}_{x} x^{r}\right)=\widetilde{p}_{x} x^{r}-\widetilde{p}_{x}^{2} x^{r}=0, \\
x \widetilde{p}_{x}=x p_{x}\left(e_{a}-y x\right)=x p_{x}-\left(x p_{x}\right) y x=0, \\
x \widetilde{x}_{r}=x\left(x^{r}-\widetilde{p}_{x} x^{r}\right)=x x^{r}-\left(x \widetilde{p}_{x}\right) x^{r}=e_{a}-0=e_{a},
\end{gathered}
$$

i.e. $\widetilde{x}^{r}$ is a right inverse of $x$. The morphism $\widetilde{p}_{x}$ is an idempotent. Indeed,

$$
\begin{aligned}
\widetilde{p}_{x}^{2} & =\left[p_{x}\left(e_{a}-y x\right)\right]^{2}=\left(p_{x}-p_{x} y x\right)^{2}=p_{x}^{2}-p_{x}^{2} y x-p_{x} y x p_{x}+\left(p_{x} y x\right)\left(p_{x} y x\right) \\
& =p_{x}-\left(p_{x} y\right) x-\left(p_{x} y\right)\left(x p_{x}\right)+\left(p_{x} y\right)\left(x p_{x} y x\right)=p_{x} .
\end{aligned}
$$

Definition 2.1. A pararing $P$ has the Kaplansky-Jacobson property if $P$ has units and the existence of a right inverse of a morphism $x \in P$ implies the existence of infinitely many right inverses. A pararing with this property will be called briefly a $K$-J-pararing. A $K$-J-paraalgebra is a paraalgebra which, as a pararing, is a K-J-pararing.

This definition and Corollary 2.1 immediately imply

COROLlaRY 2.2. Every pararing with units has the Kaplansky-Jacobson property.

COROLLARY 2.3. Every irreducible pararing with units is a ring with the Kaplansky-Jacobson property.

COROLLARY 2.4. Every paraalgebra has the Kaplansky-Jacobson property.

In a similar manner we obtain dual results for left invertible operators:

Theorem 2.3. Let $P$ be a pararing with units. Let $x \in P$ be left invertible and let $x^{l}$ be its left inverse. Then there is an $a \in P$ such that the morphism $q_{x}=e_{a}-x x^{l}$ is well defined and $q_{x} \in M_{a \mid a}$. Moreover, $q_{x}$ is an idempotent such that $q_{x} x=0$ and $x^{l} q_{x}=0$.

$\left({ }^{1}\right)$ Cf. Jacobson $[J 1]$. 
Corollary 2.5. Let $P$ be a pararing with units. Let $x \in P$ be left invertible and let $x^{l}$ be its left inverse, i.e. there is an $a \in P$ such that the idempotent $q_{x}=e_{a}-x x^{l} \in M_{a \mid a}$. Then every left inverse of $x$ is of the form $x_{1}^{l}=x^{l}+y q_{x}$, where $y q_{x}$ exists and belongs to $M_{a \mid a}$. In particular, $x_{k}^{l}=x^{k} q p_{x}$ for $k \in \mathbb{N}$ are right inverses of $x$.

TheOREM 2.4. Let $P$ be a pararing with units. Let $x \in P$ be left invertible and let $q_{x}=e_{a}-x x^{l} \in M_{a \mid a}$ be an idempotent corresponding to a left inverse $x^{l}$ of $x\left(a \in M_{a \mid a} \subset P\right)$. Whenever $x y$ exists and belongs to $M_{a \mid a}$, the morphism $\widetilde{q}_{x}=\left(e_{a}-x y\right) q_{x}$ is an idempotent corresponding to a left inverse $\widetilde{x}^{l}=x^{l}-x \widetilde{q}_{x}$ of $x$ such that $\widetilde{x}^{l} \widetilde{q}_{x}=0$ and $\widetilde{q}_{x} x=0$.

Definition 2.2. A pararing $P$ has the dual Kaplansky-Jacobson property if $P$ has units and the existence of a left inverse of a morphism $x \in P$ implies the existence of infinitely many left inverses. A pararing with this property will be called briefly a dual K-J-pararing. A dual K-J-paraalgebra is a paraalgebra which, as a pararing, is a dual K-J-pararing.

This definition and Corollary 2.5 immediately imply

COROLlary 2.6. Every pararing with units has the dual Kaplansky-Jacobson property.

COROLLARY 2.7. Every irreducible pararing with units is a ring with the dual Kaplansky-Jacobson property.

Corollary 2.8. Every paraalgebra has the dual Kaplansky-Jacobson property.

If the projectors $p_{x}$ (respectively, $q_{x}$ ) are well defined for some $x \in P$ then they induce partitions of units. Namely, every morphism of the form $e_{a}-p_{x}=x^{r} x$ (respectively, $e_{a}-q_{x}=x x^{l}$ ) is also an idempotent and $x y$ exists $\Rightarrow y=p_{x} y+\left(e_{a}-p_{x}\right) y$ (resp. $z x$ exists $\Rightarrow z=z q_{x}+z\left(e_{a}-q_{x}\right)$ ).

Theorem 2.5. Let $P$ be a $K-J$ pararing. Let $x \in P$ be right (left) invertible and let $p_{x}=e_{a}-x^{r} x \in M_{a \mid a}$ (respectively, $\left.q_{x}=e_{a}-x x^{l}\right)$ be an idempotent corresponding to a right inverse $x^{r}$ (respectively, a left inverse $\left.x^{l}\right)$ of $x\left(a \in M_{a \mid a} \subset P\right)$. Whenever $x+y$ exists and belongs to $M_{a \mid a}$, the morphism $y$ is called an $x$-perturbation if $x+y$ is again right (left) invertible. Then all morphisms of the form $y=x^{\prime} p_{x}$ (respectively, $y=q_{x} x^{\prime}$, if they exist) are $x$-perturbations.

Proof. By Theorem 2.4, $(x+y) x^{r}=x x^{r}+\left(x^{\prime} p_{x}\right) x^{r}=e_{a}+x^{\prime}\left(p_{x} x^{r}\right)=e_{a}$. A similar proof for left invertible morphisms.

3. Algebraic Analysis in Kaplansky-Jacobson paraalgebras. Let $X$ be a linear space over a field $\mathbb{F}$ of scalars (of characteristic zero). Suppose 
that $D \in R(X)$, i.e. $D$ is a right invertible operator with $\operatorname{dom} D \subset X$ and range also in $X$. (For all notions and properties connected with right invertible operators cf. [PR3].) Note that, by definition, $D$ is a surjective mapping.

Write $\mathbb{N}_{0}=\{0\} \cup \mathbb{N}, \mathbb{N}_{\infty}=\{0,1,2, \ldots,+\infty\}$ and

$$
D_{0}=X, \quad D_{k}=\operatorname{dom} D^{k} \quad \text { for } k \in \mathbb{N}, \quad D_{\infty}=\bigcap_{k \in \mathbb{N}_{0}} D_{k} .
$$

Clearly,

$$
D_{\infty} \subset \cdots \subset D_{k}=\operatorname{dom} D^{k} \subset \cdots \subset D_{1}=\operatorname{dom} D \subset D_{0}=X
$$

and $D_{j} \neq D_{k}$ if $\operatorname{dom} D \neq X\left(j, k \in \mathbb{N}_{\infty}\right)$.

For a given $D \in R(X)$ we denote by $I$ the identity operator and

- $\operatorname{ker} D=\{z \in \operatorname{dom} D: D z=0\}$, the kernel of $D$, also called the space of constants;

- $\mathcal{R}_{D}=\left\{R \in L_{0}(X): D R=I\right\}$, the set of right inverses of $D$;

- $\mathcal{F}_{D}=\left\{F \in L_{0}(X): F^{2}=F, F X=\operatorname{ker} D, \exists_{R \in \mathcal{R}_{D}} F R=0\right\}$, the set of initial operators for $D$.

We have $\operatorname{dom} D=R X \oplus \operatorname{ker} D$ for all $R \in \mathcal{R}_{D}$.

$F$ is an initial operator for $D \in R(X)$ corresponding to an $R \in \mathcal{R}_{D}$, i.e., $F \in \mathcal{F}_{D}$, if and only if

$$
F=I-R D \quad \text { on } \operatorname{dom} D .
$$

Even more, any projection $F^{\prime}$ onto ker $D$ is an initial operator for $D$ corresponding to a right inverse $R^{\prime}=R-F R^{\prime}$, for any $R \in \mathcal{R}_{D}$.

If we know at least one right inverse $R$ then we also have the general forms of the sets of right inverses and initial operators for a right invertible operator $D$. Namely,

$$
\mathcal{R}_{D}=\left\{R+F A: A \in L_{0}(X)\right\}, \quad \mathcal{F}_{D}=\left\{F(I-A D): A \in L_{0}(X)\right\},
$$

where $F$ is any initial operator for $D$ corresponding to $R$ (cf. Theorem 2.1 and Corollary 2.1).

If two right inverses (respectively, initial operators) commute with each other, then they are equal.

Write $\mathcal{R}_{D}=\left\{R_{\gamma}\right\}_{\gamma \in \Gamma}$. Then, by (3.2), $\mathcal{R}_{D}$ induces the family $\mathcal{F}_{D}=$ $\left\{F_{\gamma}\right\}_{\gamma \in \Gamma}$ of initial operators defined by

$$
F_{\gamma}=I-R_{\gamma} D \quad \text { on } \operatorname{dom} D \quad(\gamma \in \Gamma) .
$$

Formula (3.3) yields (by a two-lines induction) the Taylor-Gontcharov Formula, which plays a fundamental role in our theory. Namely, let $\left\{\gamma_{n}\right\} \subset \Gamma$ be 
an arbitrary sequence. Then for all positive integers $N$ the following identity holds:

$$
\begin{aligned}
I=F_{\gamma_{0}}+\sum_{k=0}^{N-1} R_{\gamma_{0}} \cdots R_{\gamma_{k-1}} F_{\gamma_{k}} D^{k}+R_{\gamma_{0}} \cdots R_{\gamma_{N-1}} D^{N} \\
\quad \text { on } \operatorname{dom} D^{N}(N \in \mathbb{N}) .
\end{aligned}
$$

Let $F$ be an initial operator for $D$ corresponding to an $R \in \mathcal{R}_{D}$. Putting in (3.4) $R_{\gamma_{n}}=R$ and $F_{\gamma_{n}}=F(n \in \mathbb{N})$, we obtain the Taylor Formula:

$$
I=\sum_{k=0}^{n-1} R^{k} F D^{k}+R^{n} D^{n} \quad \text { on } \operatorname{dom} D^{n}(n \in \mathbb{N}) .
$$

Formula (3.5) gives partitions of units, since the operators $R^{k} F D^{k}(k=$ $0,1, \ldots, n-1), R^{n} D^{n}$ are projections onto the kernel of $D^{n}$. Thus it is easy to see the following

THEOREM 3.1. The paraalgebra

$$
\mathcal{D}\left(D_{k} ; \mathbb{N}_{\infty}\right)=L_{0}\left(D_{k} \rightleftharpoons D_{j} ; j, k \in \mathbb{N}_{\infty}\right)
$$

has the Kaplansky-Jacobson property (cf. Corollary 2.4).

Theorem 2.5 immediately implies

Theorem 3.2. Let $A \in \mathcal{D}\left(D_{k} ; \mathbb{N}_{\infty}\right)$. Then $A$ is a D-perturbation if $A=T F$, where $\operatorname{dom} T \supset \operatorname{ker} D$ and $F$ is an initial operator for $D$ corresponding to a right inverse $R$.

Corollary 3.1. Suppose that $J$ is a quasi Fredholm ideal in the paraalgebra $\mathcal{D}\left(D_{k} ; \mathbb{N}_{\infty}\right)$. Then

$$
J \mathcal{F}_{D}=\left\{T F \in \mathcal{F}_{D}: T \in J, \operatorname{dom} D \supset \operatorname{ker} D\right\} \subset J,
$$

i.e. if $A=T F$ is a D-perturbation with $T \in J$ then $T F \in J$ and the operator $I+T F$ has a finite d-characteristic.

The following question arises: Are all $D$-perturbations of the form $T F$ ?

Suppose that $D_{1}, \ldots, D_{m} \in R(X)$ and that the superposition $D=$ $D_{1} \cdots D_{m}$ is well defined. Let $F_{j}$ be an initial operator for $D_{j}$ corresponding to an $R_{j} \in \mathcal{R}_{D_{j}}(j=1, \ldots, m)$. Write

$$
R=R_{m} \cdots R_{1}, \quad F=F_{m}+R_{m} F_{m-1} D_{m}+\cdots+R_{m} \cdots R_{2} F_{1} D_{2} \cdots D_{m} .
$$

Then $D \in R(X), R \in \mathcal{R}_{D}$ and $F$ is an initial operator for $D$ corresponding to $R$.

If $X$ is an algebra, $D \in R(X)$ and $x y, y x \in \operatorname{dom} D$ whenever $x, y \in$ dom $D$ then we write $D \in \mathbf{A}(X)$. If $X$ is a commutative algebra and $D \in$ $\mathbf{A}(X)$ then we write $D \in \mathrm{A}(X)$. If $D \in \mathbf{A}(X)$ then we may write

$$
D(x y)=c_{D}[x D y+(D x) y]+f_{D}(x, y) \quad \text { for } x, y \in \operatorname{dom} D,
$$


where $c_{D}$ is a scalar depending on $D$ only, $f_{D}$ is called the non-Leibniz component and $f_{D}$ (by its definition) is a bilinear mapping of $\operatorname{dom} D \times \operatorname{dom} D$ into $X$. In commutative algebras the mapping $f_{D}$ is symmetric. If $c_{D}=1$ and $f_{D}=0$ then $X$ is said to be a Leibniz algebra, because

$$
D(x y)=x D y+(D x) y \quad \text { for } x, y \in \operatorname{dom} D .
$$

In commutative Leibniz algebras the generalized Leibniz formula holds:

$$
D^{k}(x y)=c_{D}^{k}\left(x D^{k} y+y D^{k} x\right)+f_{D}^{k}(x, y) \quad \text { for } x, y \in D_{k}(k \in \mathbb{N}),
$$

where

$$
f_{D}^{1}=f_{D}=0, \quad f_{D}^{k}(x, y)=\sum_{j=1}^{k-1}\left(\begin{array}{c}
k \\
j
\end{array}\right)\left(D^{k-j} x\right)\left(D^{j} y\right) \quad \text { for } k \geq 2 .
$$

Definition 3.1. Suppose that $D \in R(X)$. The paraalgebra $\mathcal{D}\left(D_{k} ; \mathbb{N}_{\infty}\right)$ $=L_{0}\left(D_{k} \rightleftharpoons D_{j} ; j, k \in \mathbb{N}_{\infty}\right)$ is said to be a D-paraalgebra if $D \in \mathbf{A}(X)$. The paraalgebra $\mathcal{D}\left(D_{k} ; \mathbb{N}_{\infty}\right)$ is a Leibniz paraalgebra if $X$ is a Leibniz algebra.

Clearly, if $\operatorname{dom} D=X$ then $\mathcal{D}\left(D_{k} ; \mathbb{N}_{\infty}\right)$ is an algebra, since

$$
\mathcal{D}\left(D_{k} ; \mathbb{N}_{\infty}\right)=L_{0}\left(D_{k} \rightleftharpoons D_{j} ; j, k \in \mathbb{N}_{\infty}\right)=L_{0}(X \rightarrow X)=L_{0}(X) .
$$

Note that the multiplication in $X$ is not necessarily commutative.

Some results can also be proved for left invertible operators. Write

- $\Lambda(X)$ is the set of all left invertible operators belonging to $L_{0}(X)$ (we assume that $\operatorname{dom} T=X$ for $T \in \Lambda(X))$;

- $\mathcal{L}_{T}=\{S \in L(X): S T=I\}$ is the set of all left inverses to $T \in \Lambda(X)$;

- $\mathcal{G}_{T}=\left\{G \in L(X): G^{2}=G\right.$, $\left.\operatorname{ker} G=T X, \exists_{S \in \mathcal{L}_{T}} S Q=0\right\}$ is the set of all co-initial operators for $T \in \Lambda(X)$ (cf. Theorem 2.4 and Corollary 2.8).

- $\mathcal{I}(X)=R(X) \cap \Lambda(X)$ is the set of invertible operators $\left({ }^{1}\right)$.

Clearly, if ker $D \neq\{0\}$, then the operator $D$ is right invertible, but not invertible. Here the invertibility of an operator $A \in L(X)$ means that the equation $A x=y$ has a unique solution for every $y \in X$. If $D \in \mathcal{I}(X)$ then $\mathcal{F}_{D}=\mathcal{G}_{D}=\{0\}$ and $\mathcal{R}_{D}=\mathcal{L}_{D}=\left\{D^{-1}\right\}$.

The sets defined above are, in a sense, dual. Namely, if $D \in R(X)$ and $F \in \mathcal{F}_{D}$ corresponds to an $R \in \mathcal{R}_{D}$, i.e. $F R=0$, then $R \in \Lambda(X)$ and $F \in \mathcal{G}_{D}$ corresponds to $D \in \mathcal{L}_{D}$.

With these facts one can obtain Calculus and solutions to linear equations (under appropriate assumptions on the equations considered). If the

$\left({ }^{1}\right)$ Note that the domain of a left invertible operator is the whole space $X$, so, in this case, instead of a paraalgebra we have an algebra. 
field $\mathbb{F}$ is algebraically closed then solutions of linear equations with scalar coefficients can be calculated by a decomposition of a rational function into vulgar fractions (as in Operational Calculus).

Recall that any solution of the equation

$$
D x=y, \quad y \in X, D \in R(X),
$$

is of the form $x=R y+z$, where $R \in \mathcal{R}_{D}$ and $z \in \operatorname{ker} D$ is arbitrary. This form is independent of the choice of $R$. Indeed, if $R^{\prime} \in \mathcal{R}_{D}$ and $R^{\prime} \neq R$ then for all $x \in X$ we have $R^{\prime} x-R x \in \operatorname{ker} D$. So a change of $R$ implies only a change of the constant $z$.

The equation

$$
T x=y, \quad y \in T X, T \in \Lambda(X),
$$

has a unique solution $x=S y$ which is independent of the choice of $S \in \mathcal{L}_{T}$. Indeed, by definition, $x=S T x=S y$ and $\operatorname{ker} T=\{0\}$. Write

$$
Q(D)=\sum_{k=0}^{N} Q_{k} D^{k}, \quad \text { where } D \in R(X), Q_{0}, \ldots, Q_{N-1} \in L(X), Q_{N}=I .
$$

If there is an $R \in \mathcal{R}_{D}$ such that the operator

$$
Q(I, R)=\sum_{k=0}^{N} Q_{k} R^{N-k}
$$

is invertible, then $Q(D) \in R(X)$ and $R^{N}[Q(I, R)]^{-1} \in \mathcal{R}_{Q(D)}$. Thus an initial value problem for $Q(D)$, that is, the problem of finding solutions of the equation $Q(D) x=y, y \in X$, with the initial conditions

$$
F D^{k} x=y_{k}, \quad \text { where } y_{k} \in \operatorname{ker} D \text { are given }(k=0,1, \ldots, N-1),
$$

has for every $y, y_{0}, \ldots, y_{N-1}$ a unique solution of the form

$$
x=R^{N}[Q(I, R)]^{-1}\left[y-\sum_{m=0}^{N-1}\left(\sum_{k=0}^{m} Q_{k} R^{m-k}\right) y_{m}\right]+\sum_{k=0}^{N-1} R^{k} y_{k},
$$

i.e. this problem is well-posed.

An $A \in L_{0}(X)$ is said to be a Volterra operator if the operators $I-\lambda A$ are invertible for every $\lambda \in \mathbb{F}$, i.e. if for every $\lambda \in \mathbb{F} \backslash\{0\}$ the number $1 / \lambda$ is a regular value of $A$. The set of all Volterra operators belonging to $L(X)$ will be denoted by $V(X)$.

Main advantages of Algebraic Analysis are: (i) simplifications of proofs due to an algebraic description of problems under consideration; (ii) algorithms for solving "similar" problems, although these similarities could be rather far from one another and very formal, and (iii) several new results even in the classical case of the operator $d / d t$, which was, indeed, unexpected 
(for various extensions of the above results, examples and applications cf. [PR3]-[PR13], [PRW1], [PRW2], [M1], [M2], [N2], [T], [V], and others).

There are several applications to ordinary and partial differential equations with scalar and variable coefficients, functional-differential equations and discrete analogues of these equations, for instance, difference equations. There are also some results for non-linear equations.

It should be pointed out that in Algebraic Analysis a notion of convolution is not necessary. Also there is no need to have a structure of the Mikusiński field. This, together with the noncommutativity of right inverses and initial operators, shows the essential distinction of Algebraic Analysis from Operational Calculus.

ExAmple 3.1. Let $X=C[0,1]$ over $\mathbb{F}$, where $\mathbb{F}=\mathbb{R}$ or $\mathbb{C}$, and let $D=$ $d / d t$. Then $\operatorname{ker} D$ consists of all constant functions. The operators $R_{a}=\int_{a}^{t}$, $a \in[0,1]$, are Volterra right inverses of $D$. Observe that $\operatorname{dim} \operatorname{ker} D=1$. The family of initial operators induced by $R_{a}$ is defined as follows: $\left(F_{a} x\right)(t)=$ $x(a)$ for $x \in X$ and $a \in[0,1]$. Consider operators of the form

$$
\begin{gathered}
\left(\widetilde{F}_{a} x\right)(t)=\frac{\int_{a}^{1} m(s) x(s) d s}{\int_{a}^{1} m(s) d s} \quad \text { for } x \in X, a \in[0,1], m \in X, \\
\lambda F_{a}+(1-\lambda) \widetilde{F}_{b}, \quad \lambda F_{a}+(1-\lambda) F_{b}, \quad \text { for } b \in[0,1], \lambda \in \mathbb{F} .
\end{gathered}
$$

These are also initial operators, because they are projections onto the space of constants. In several particular cases the corresponding right inverses have eigenvalues.

If we consider the space $C[0,1]$ over $\mathbb{C}$ then the only continuous Volterra right inverses are $\int_{a}^{t}, a \in[0,1]$. However, if $\mathbb{F}=\mathbb{R}$, then one can find a Volterra right inverse which is not of that form (cf. [PRR2], [PRR3]).

The Taylor formula applied to a function $x \in \operatorname{dom} D^{N}=C^{N}[0,1]$ gives a classical Taylor formula with remainder in integral form. In order to obtain remainders either in the Lagrange form or in the Cauchy form it is enough to apply the Darboux property of continuous functions satisfying two different estimates, without any intermediate value theorem, like the Rolle theorem or Lagrange theorem.

Observe that $X=C[0,1]$ is a commutative algebra (a linear ring) with respect to the usual pointwise multiplication. The operator $D$ satisfies the so-called Leibniz condition:

$$
D(x y)=x D y+y D x \quad \text { for } x, y \in \operatorname{dom} D=C^{1}[0,1] .
$$

By this condition, $\mathcal{D}\left(C^{k}[0,1] ; \mathbb{N}_{\infty}\right)$ is a Leibniz paraalgebra.

We point out that the restriction to the interval $[0,1]$ is not essential. The same considerations are valid also for any interval $[a, b]$ and for a half-axis or the real line. 
ExAmple 3.2. Let $X=C[0, T]$ with multiplication defined by convolution $*$ and let $D=d / d t$. Then $X$ is not a Leibniz algebra, for the following Duhamel condition holds:

$$
\begin{aligned}
\frac{d}{d t}(x * y)(t)=\left(x * \frac{d}{d t}\right)(t)+x(t) y(0) & -x(0) y(t), \\
x & \in C[0, T), y \in C^{1}(0, T) .
\end{aligned}
$$

If we write a similar formula for $y * x$ then, by commutativity, we find

$$
x * y=\frac{1}{2}(x * y+y * x)=\frac{1}{2}\left(x * \frac{d}{d t}+\frac{d}{d t} x * y\right),
$$

which implies that the non-Leibniz component $f_{D}$ is 0 , but $c_{D}=\frac{1}{2}$. Thus $\mathcal{D}\left(C^{k}[0, T] ; k \in \mathbb{N}_{\infty}\right)$ is a paraalgebra, but it is not a Leibniz paraalgebra.

Note that in both examples we have $\operatorname{dom} D \subset X$ but $\operatorname{dom} D \neq X$. We should point out that in order to solve some problems, it is necessary to assume that the field $\mathbb{F}$ of scalars under consideration is algebraically closed. Clearly, if $\mathbb{F}=\mathbb{R}$, we may consider a natural extension to $\mathbb{C}$.

4. Logarithms and antilogarithms in paraalgebras with the Kaplansky-Jacobson property. Let $X$ be an algebra (over a field of scalars $\mathbb{F}$ with characteristic zero) and let $D \in \mathbf{A}(X)$. As in the previous section, consider the paraalgebra

$$
\mathcal{D}\left(D_{k} ; \mathbb{N}_{\infty}\right)=L_{0}\left(D_{k} \rightleftharpoons D_{j} ; j, k \in \mathbb{N}_{\infty}\right),
$$

which, by Corollary 2.4, has the Kaplansky-Jacobson property. Write $I(X)$ for the set of all invertible elements in $X$.

Lemma 4.1. Suppose that $a \in \mathcal{D}\left(D_{k} ; \mathbb{N}_{\infty}\right), a \in M_{a \mid a}$ and $u, D x \in$ $M_{a \mid a} \cap \operatorname{dom} D$. Then $u D x,(D x) u$ exist and belong to $M_{a \mid a}$.

Proof. By the axiom $\mathbf{A}_{1}$, the existence of $u=u e_{a}, e_{a}=e_{a}^{2}, e_{a} D x$ implies the existence of $u D x$. Moreover, $a(u D x) a=(a u)[(D x)] a$, i.e. $u D x \in M_{a \mid a}$. A similar proof for $(D x) u$.

Definition 4.1. Suppose that $a \in \mathcal{D}\left(D_{k} ; \mathbb{N}_{\infty}\right)$ and $u, D x \in M_{a \mid a} \cap$ $\operatorname{dom} D$ for all $a$ such that $a \in M_{a \mid a}$. Let $\Omega_{r}, \Omega_{l}: \operatorname{dom} D \rightarrow 2^{\operatorname{dom} D}$ be multifunctions defined as follows: for $u \in \operatorname{dom} D$,

$$
\begin{aligned}
& \Omega_{r} u=\{x \in \operatorname{dom} D: u D x \text { exists } \Rightarrow D u=u D x\}, \\
& \Omega_{l} u=\{x \in \operatorname{dom} D:(D x) u \text { exists } \Rightarrow D u=(D x) u\} .
\end{aligned}
$$

The equations

$$
\begin{array}{ll}
D u=u D x & \text { for }(u, x) \in \operatorname{graph} \Omega_{r}, \\
D u=(D x) u & \text { for }(u, x) \in \operatorname{graph} \Omega_{l}
\end{array}
$$


are said to be the right and left basic equations, respectively. Clearly,

$$
\begin{aligned}
& \Omega_{r}^{-1} x=\{u \in \operatorname{dom} D: u D x \text { exists } \Rightarrow D u=u D x\}, \\
& \Omega_{l}^{-1} x=\{u \in \operatorname{dom} D:(D x) u \text { exists } \Rightarrow D u=(D x) u\}
\end{aligned}
$$

for $x \in \operatorname{dom} D$.

If $D \in \mathrm{A}(X)$ then $\Omega_{r}=\Omega_{l}$ and we write $\Omega_{r}=\Omega_{l}=\Omega$. Clearly, in this case we have $\operatorname{dom} \Omega_{r}=\operatorname{dom} \Omega_{l}=\operatorname{dom} \Omega$.

The multifunctions $\Omega_{r}, \Omega_{l}, \Omega$ are well defined. If $D$ is right (left) invertible or invertible, their domains are non-empty (cf. [PR4] for the case of algebras). By definition, if $x, y \in \Omega_{r}$ (respectively, $\Omega_{l}, \Omega$ ) then $x-y \in \operatorname{ker} D$, i.e. it is a constant. Moreover, these multifunctions map $\operatorname{ker} D$ into itself.

Suppose that $\left(u_{r}, x_{r}\right) \in$ graph $\Omega_{r},\left(u_{l}, x_{l}\right) \in$ graph $\Omega_{l}, L_{r}, L_{l}$ are selectors of $\Omega_{r}, \Omega_{l}$, respectively, and $E_{r}, E_{l}$ are selectors of $\Omega_{r}^{-1}, \Omega_{l}^{-1}$, respectively. By definition, $L_{r} u_{r} \in \operatorname{dom} \Omega_{r}^{-1}, E_{r} x_{r} \in \operatorname{dom} \Omega_{r}, L_{l} u_{l} \in \operatorname{dom} \Omega_{l}^{-1}$, $E_{l} x_{l} \in \operatorname{dom} \Omega_{l}$ and the following equations are satisfied:

$$
\begin{aligned}
D u_{r} & =u_{r} D L_{r} u_{r}, \quad D E_{r} x_{r}=\left(E_{r} x_{r}\right) D x_{r} ; \\
D u_{l} & =\left(D L_{l} u_{l}\right) u_{l}, \quad D E_{l} x_{l}=\left(D x_{l}\right) E_{l} x_{l} .
\end{aligned}
$$

Let $F$ be an initial operator for $D$ corresponding to an $R \in \mathcal{R}_{D}$ and let $u_{r}, u_{l} \in I(X)$. Then these equations can be written in equivalent forms

$$
\begin{aligned}
L_{r} u_{r} & =R\left[u_{r}^{-1} D u_{r}\right]+F L_{r} u_{r}, & E_{r} x_{r}-R\left[\left(E_{r} x_{r}\right)\left(D x_{r}\right)\right] & =F E_{r} x_{r} ; \\
L_{l} u_{l} & =R\left[\left(D u_{l}\right) u_{l}^{-1}\right]+F L_{l} u_{l}, & {\left[I-R\left(D x_{l}\right)\right] E_{l} x_{l} } & =F E_{l} x_{l} .
\end{aligned}
$$

Definition 4.2. Any invertible selector $L_{r}$ of $\Omega_{r}$ is said to be a right logarithmic mapping and its inverse $E_{r}=L_{r}^{-1}$ is said to be a right antilogarithmic mapping. If $\left(u_{r}, x_{r}\right) \in$ graph $\Omega_{r}$ and $L_{r}$ is an invertible selector of $\Omega_{r}$ then the element $L_{r} u_{r}$ is said to be a right logarithm of $u_{r}$ and $E_{r} x_{r}$ is said to be a right antilogarithm of $x_{r}$. By $G\left[\Omega_{r}\right]$ we denote the set of all pairs $\left(L_{r}, E_{r}\right)$, where $L_{r}$ is an invertible selector of $\Omega_{r}$ and $E_{r}=L_{r}^{-1}$. Similarly, any invertible selector $L_{l}$ of $\Omega_{l}$ is said to be a left logarithmic mapping and its inverse $E_{l}=L_{l}^{-1}$ is said to be a left antilogarithmic mapping. If $\left(u_{l}, x_{l}\right) \in$ graph $\Omega_{l}$ and $L_{l}$ is an invertible selector of $\Omega_{l}$ then the element $L_{l} u$ is said to be a left logarithm of $u_{l}$ and $E_{l} x_{l}$ is said to be a left antilogarithm of $x_{l}$. By $G\left[\Omega_{l}\right]$ we denote the set of all pairs $\left(L_{l}, E_{l}\right)$, where $L_{l}$ is an invertible selector of $\Omega_{l}$ and $E_{l}=L_{l}^{-1}$.

If $D \in \mathrm{A}(X)$ then $\Omega_{l}=\Omega_{r}=\Omega$ and we write

$$
L_{r}=L_{l}=L, \quad E_{r}=E_{l}=E, \quad(L, E) \in G[\Omega] .
$$

Selectors $L, E$ of $\Omega$ are said to be logarithmic and antilogarithmic mappings, respectively. For any $(u, x) \in$ graph $\Omega,(L, E) \in G[\Omega]$ the elements $L u, E x$ are said to be the logarithm of $u$ and the antilogarithm of $x$, respectively. 
Note that in the paraalgebra defined in Example 3.2 invertible selectors of the multifunction $\Omega$, hence also logarithms, do not exist.

Clearly, by definition, for all $\left(L_{r}, E_{r}\right) \in G\left[\Omega_{r}\right],\left(u_{r}, x_{r}\right) \in$ graph $\Omega_{r}$, $\left(L_{l}, E_{l}\right) \in G\left[\Omega_{l}\right],\left(u_{l}, x_{l}\right) \in$ graph $\Omega_{l}$ we have

$$
\begin{gathered}
E_{r} L_{r} u_{r}=u_{r}, \quad L_{r} E_{r} x_{r}=x_{r} ; \quad E_{l} L_{l} u_{l}=u_{l}, \quad L_{l} E_{l} x_{l}=x_{l} ; \\
D E_{r} x_{r}=\left(E_{r} x_{r}\right) D x_{r}, \quad D u_{r}=u_{r} D L_{r} u_{r} ; \\
D E_{l} x_{l}=\left(D x_{l}\right)\left(E_{l} x_{l}\right), \quad D u_{l}=\left(D L_{l} u_{l}\right) u_{l} .
\end{gathered}
$$

Moreover,

$$
u D L_{r} u-\left(D L_{l} u\right) u=0 \quad \text { for all } u \in \operatorname{dom} \Omega_{r} \cap \operatorname{dom} \Omega_{l} .
$$

If $D \in R(X)$ (respectively, $\Lambda(X), I(X)$ ) then the right (left) logarithm of zero is not defined. On the other hand, $E_{r}(0), E_{l}(0), E(0) \in \operatorname{ker} D$.

Definition 4.3. A right logarithmic mapping $L_{r}$ (left logarithmic mapping $L_{l}$, logarithmic mapping $L$, respectively) is said to be of the exponential type if whenever $u v$ exists, then

$$
\begin{aligned}
& L_{r}(u v)=L_{r} u+L_{r} v \quad \text { for } u, v \in \operatorname{dom} \Omega_{r} ; \\
& L_{l}(u v)=L_{l} u+L_{l} v \quad \text { for } u, v \in \operatorname{dom} \Omega_{l} \text {; } \\
& L(u v)=L u+L v \quad \text { for } u, v \in \operatorname{dom} \Omega \text {, }
\end{aligned}
$$

respectively.

Theorem 4.1. Let $D \in R(X)$.

(i) If $D \in \mathbf{A}(X),\left(L_{r}, E_{r}\right) \in G\left[\Omega_{r}\right],\left(L_{l}, E_{l}\right) \in G\left[\Omega_{l}\right]$ and $L_{r}\left(L_{l}\right.$, respectively) is of the exponential type then

$$
E_{r}(x+y)=\left(E_{r} x\right)\left(E_{r} y\right), \quad E_{l}(x+y)=\left(E_{l} x\right)\left(E_{l} y\right),
$$

whenever $x, y \in \operatorname{dom} \Omega_{r}^{-1}\left(x, y \in \operatorname{dom} \Omega_{l}^{-1}\right.$, respectively);

(ii) if $D \in \mathrm{A}(X),(L, E) \in G[\Omega]$ and $L$ is of the exponential type then

$$
E(x+y)=(E x)(E y) \quad \text { for } x, y \in \operatorname{dom} \Omega^{-1} ;
$$

(iii) if $L_{r} \in M(X)\left({ }^{1}\right)$ then $E_{r} \in M(X)$, if $L_{l} \in M(X)$ then $E_{l} \in$ $M(X)$, if $L \in M(X)$ then $E \in M(X)$;

(iv) if $D \in \mathrm{A}(X) \cap M L(X)$ then $L, E \in M(X)$.

By Theorem 4.1(ii), if left (right) logarithmic mappings are of the exponential type, then the corresponding left (right) antilogarithmic mappings

$\left({ }^{1}\right)$ By $M(X)$ we denote the class of all multiplicative mappings of $X$ into itself, i.e. mappings $A$ such that $A(x y)=(A x)(A y)$ whenever $x y$ and $(A x)(A y)$ exist. 
satisfy the same functional equation as the usual exponential functions $c e^{\alpha t}$, $c, \alpha \in \mathbb{R}$ (cf. M. Kuczma $[\mathrm{K}])$ ). A similar conclusion for logarithmic mappings.

Definition 4.4. Let $D \in \mathbf{A}(X)$. By $\mathbf{L g}_{r}(D)\left(\mathbf{L g}_{l}(D)\right.$, respectively $)$ we denote the class of those $D$-paraalgebras with units $e_{a} \in \operatorname{dom} \Omega_{r}$ ( $e \in \operatorname{dom} \Omega_{l}$, respectively), $a \in M_{a \mid a}$, for which there exist invertible selectors of $\Omega_{r}\left(\Omega_{l}\right.$, respectively), i.e. there exist $\left(L_{r}, E_{r}\right) \in G\left[\Omega_{r}\right]\left(\left(L_{l}, E_{l}\right) \in G\left[\Omega_{l}\right]\right.$, respectively). If $D \in \mathrm{A}(X)$ then $\mathbf{L g}_{r}(D)=\mathbf{L g}_{l}(D)$. This class is denoted by $\operatorname{Lg}(D)$.

Theorem 4.2. Let either $X \in \mathbf{L g}_{r}(D)$ or $X \in \mathbf{L g}_{l}(D)$. Let $R \in \mathcal{R}_{D}$ and $g=R e$. Then $g \in \operatorname{dom} \Omega_{r}\left(g \in \operatorname{dom} \Omega_{l}, g \in \operatorname{dom} \Omega\right.$, respectively) if $g \in I(X)$. In other words: a right logarithm (left logarithm, logarithm) of $g=$ Re exists if $g$ is invertible.

Theorem 4.3. Let $X \in \mathbf{L g}_{r}(D)\left(X \in \mathbf{L g}_{l}(D), X \in \mathbf{L g}(D)\right.$, respectively). Then right (left) logarithms and antilogarithms (logarithms and antilogarithms, respectively) are uniquely determined up to a constant. These constants are additive for right (left) logarithms and logarithms and multiplicative for right (left) antilogarithms and antilogarithms.

Theorem 4.4. Suppose that $D \in \mathrm{A}(X), X \in \mathbf{L g}(D)$ and $(L, E) \in G[\Omega]$. Then $L$ is of the exponential type if and only if $X$ is a Leibniz algebra.

In other words: In commutative algebras with unit the Leibniz condition is a necessary and sufficient condition for logarithms to be of the exponential type. Thus, by Proposition 2.6, the Leibniz condition is a necessary and sufficient condition for the corresponding antilogarithms to satisfy the classical functional equation for exponential functions: $E(x+y)=(E x)(E y)$ whenever $x, y \in \operatorname{dom} \Omega^{-1} \quad$ (cf. Theorem 4.2).

Theorem 4.4 motivates the use of the name antilogarithmic mapping for the mapping inverse to a logarithmic mapping, since, in general, antilogarithmic mappings are not exponentials.

Theorem 4.5. Suppose that $D \in R(X)$ and $X \in \mathbf{L g}(D)$ is a Leibniz algebra with unit $e$. Let $u \in \operatorname{dom} D$. Then $u \in I(X)$ if and only if $u \in$ $\operatorname{dom} \Omega$.

In other words: An essential property of Leibniz commutative algebras with right invertible operators is that their elements have logarithms if and only if they are invertible. Note that in noncommutative algebras (hence also paraalgebras) Theorem 4.5 does not hold, as shown by a counterexample of A. Di Bucchianico (cf. [DB1], [DB2], also [PR4]).

Proofs are similar to those of [PR4] and following papers (cf. [PR7][PR13]). 


\section{References}

[B] W. Bitzer, Inverses in rings with identity, Amer. Math. Monthy 70 (1963), 315.

[Bra] H. Brandt, Über eine Verallgemeinerung des Gruppenbegriffes, Math. Ann. 96 (1927), 360-366.

[Bru] R. H. Bruck, A Survey of Binary Systems, Springer, Berlin, 1966.

[DB1] A. Di Bucchianico, Banach algebras, logarithms and polynomials of convolution type, J. Math. Anal. Appl. 156 (1991), 253-273.

[DB2] - Polynomials of Convolution Type, Ph.D. Thesis, Groningen, 1991.

[F] K. H. Förster, Über Kommutatoren und Störungsmetriken und ihre Beziehung zum Funktionalkalkül, Manuscripta Math. 6 (1972), 291-308.

[J1] N. Jacobson, Some remarks on one-sided inverses, Proc. Amer. Math. Soc. 1 (1950), 352-355.

[J2] - Structure of Rings, Amer. Math. Soc., Providence, RI, 1956.

[K] M. Kuczma, An Introduction to the Theory of Functional Equations and Inequalities. Cauchy's Equation and Jensen's Equation, PWN-Polish Sci. Publ. and the Silesian Univ., Warszawa-Katowice, 1985.

[LPR] H. Lausch and D. Przeworska-Rolewicz, Pseudocategories, paraalgebras and linear operators, Math. Nachr. 138 (1988), 67-82.

[M1] P. Multarzyński, On some right invertible operators in differential spaces, Demonstratio Math. 37 (2005), 905-920.

[M2] - On linear operators consistent with a subspace in differential spaces, ibid., to appear.

[N1] Nguyen Van Mau, Generalized algebraic elements and singular integral equations with transformed argument, Warsaw Univ. of Technology Publ., Warszawa, 1989.

[N2] - Boundary value problems and controllability of linear systems with right invertible operators, Dissertationes Math. 316 (1992).

[PR1] D. Przeworska-Rolewicz, On categories and pseudocategories, Funktsional. Anal. i Prilozhen. 3 (1969), no. 2, 93 (in Russian).

[PR2] - Pseudocategories, paraalgebras and perturbations of linear operators, preprint 60, Inst. Math., Polish Acad. Sci., Warszawa, 1973.

[PR3] -, Algebraic Analysis, PWN-Polish Sci. Publ. and D. Reidel, WarszawaDordrecht, 1988.

[PR4] - Logarithms and Antilogarithms. An Algebraic Analysis Approach, with appendix by Z. Binderman, Kluwer, Dordrecht, 1998.

[PR5] -, A priori determined solutions of linear equations, Math. Japon. 44 (1996), $395-412$.

[PR6] - Two centuries of Algebraic Analysis, in: Algebraic Analysis and Related Topics (Warszawa, 1999), Banach Center Publ. 53, Inst. Math., Polish Acad. Sci., Warszawa, 2000, 47-70.

[PR7] -, Postmodern logarithmo-technia, Comput. Math. Appl. 41 (2001), 1143-1154.

[PR8] -, Non-Leibniz algebras with logarithms do not have the trigonometric identity, in: Algebraic Analysis and Related Topics (Warszawa, 1999), Banach Center Publ. 53, Inst. Math., Polish Acad. Sci. Warszawa, 2000, 177-189.

[PR9] - Special functions as antilogarithms of second order, Fract. Calc. Appl. Math. 3 (2000), 277-296.

[PR10] -, True shifts revisited, Demonstratio Math. 34 (2001), 110-121.

[PR11] -, Riemann-Hilbert type problems with involutions in commutative algebras with logarithms, Fract. Calc. Appl. Math. 4 (2000), 193-208. 
[PR12] D. Przeworska-Rolewicz, Multidimensional Riemann-Hilbert type problems in Leibniz algebras with logarithms, Demonstratio Math. 35 (2002), 619-634.

[PR13] -, Generalized Sturm separation theorem, ibid. 36 (2003), 735-746.

[PRR1] D. Przeworska-Rolewicz and S. Rolewicz, Equations in Linear Spaces, Monogr. Mat. 47, PWN-Polish. Sci. Publ., Warszawa, 1968.

[PRR2] -, - , The only continuous Volterra right inverses in $C_{c}[0,1]$ for the operator $\frac{\mathrm{d}}{\mathrm{d} t}$ are $\int_{a}^{t}$, Colloq. Math. 51 (1987), 279-283.

[PRR3] - - - On a representation of continuous Volterra right inverses to the derivative in the space of infinitely differentiable functions, J. Austral. Math. Soc. 41 (1986), 138-142.

[PRW1] D. Przeworska-Rolewicz and H. Wysocki, An algebraic analysis approach to 2-D discrete problems, Control Cybernet. 30 (2001), 149-158.

[PRW2] -, 一, A generalization of Lagrange, du Bois-Reymond and Legendre lemmas using the Algebraic Analysis theory, Studia Sci. Math. Hungar. 41 (2000), $267-284$.

[SG] K. P. Shum and Y. Q. Guo, A new proof of Kaplansky-Jacobson theorem on one-sided inverses, Sci. Math. Japon. 59 (2004), 531-533.

[T] H. von Trotha, Structure properties of $D-R$ spaces, Dissertationes Math. 184 (1981).

[V] G. Virsik, Right inverses of vector fields, J. Austral. Math. Soc. 58 (1995), $411-420$.

Institute of Mathematics

Polish Academy of Sciences

P.O. Box 21, Śniadeckich 8

00-956 Warszawa 10, Poland

E-mail: rolewicz@impan.gov.pl 